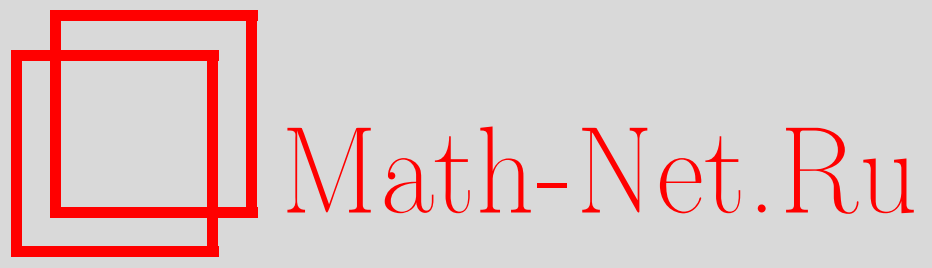

Т. Бен Зинеб, Э. Гобе, Аналитическая аппроксимация плавающих рент для малой волатильности и малой выплаты, Теория вероятн. и ее примен., 2016, том 61, выпуск 1, 5-25

DOI: https://doi.org/10.4213/tvp5041

Использование Общероссийского математического портала Math-Net.Ru подразумевает, что вы прочитали и согласны с пользовательским соглашением http://www.mathnet.ru/rus/agreement

Параметры загрузки:

IP: 54.209 .52 .79

26 апреля 2023 г., 18:11:39

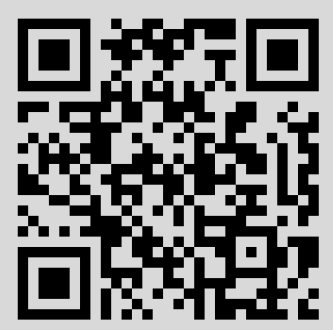




\title{
АНАЛИТИЧЕСКАЯ АППРОКСИМАЦИЯ ПЛАВАЮЩИХ РЕНТ ДЛЯ МАЛОЙ ВОЛАТИЛЬНОСТИ И МАЛОЙ ВЫПЛАТЫ ${ }^{1)}$
}

\author{
(Перевод Иванова Р.В.)
}

\begin{abstract}
В данной работе обсуждаются задачи асимптотической аппроксимации в рамках актуарной тематики. Предложен метод точных аппроксимаций размеров плавающей ренты для гарантированной минимальной компенсачионной выплаты (GMWB) в составной модели (модель Блэка-Шоулса плюс модель Халла-Уайта). С помощью этого метода получены точные достаточно простые явные формулы, которые почти мгновенно вычисляются на компьютере и в рамках наших экспериментов дают точность около $0.01 \%$. Оценки ошибок подтверждают сходимость наших аппроксимаций в случае, когда размеры выплаты и волатильности малы.
\end{abstract}

Ключевые слова и фразы: точные формулы, аналитические аппроксимации, гарантированная минимальная компенсационная выплата (GMWB), оценки ошибок.

1. Введение. В последнее десятилетие, асимптотические методы стали весьма популярными в стохастических финансах как способ получения быстро сходящихся и точных аппроксимаций для ожидаемой волатильности в моделях нахождения цен финансовых инструментов (обзор см., например, в [3] и [12]). Однако в актуарных расчетах эти методы только начинают использоваться. Цель данной работы - продемонстрировать, как принципы асимптотической аппроксимации могут работать в рассматриваемой области, несмотря на то что в страховании моделирование волатильности традиционно является простым. Это означает, что в данном случае для нахождения аппроксимаций приходится опираться на иные асимптотические параметры.

Плавающая рента традиционно используется в продуктах страхования жизни, обеспечивая клиентам защиту от инвестиционного риска и риска смертности; подробное описание развития рынка и продуктов

${ }^{*}$ Centre de Mathématiques Appliqués, École Polytechnique; CNRS, Palaiseau, France; e-mail: tarik.benzineb@gmail.com; emmanuel.gobet@polytechnique.edu

1) Исследование первого автора выполнено при поддержке AXA Fund Research и ANRT (CIFRE PhD Thesis). Исследование второго автора является частью the Chair Financial Risks of the Risk Foundation. 
страхования см., например, в [15], [8]. Ввиду большой гибкости контрактов вычисление их стоимости и хеджирование весьма затруднительны, и это все еще остается важным вопросом для страховых компаний, заслуживающим серьезных исследований.

Что касается моделирования, то в зависимости от учитываемых рисков (риски смертности, изменения процентной ставки, фондовый риск, возможность выбора дат выплат) и от модели расчетов (дискретное или непрерывное время), можно найти много работ, связанных с определением необходимых правил расчетов цен и хеджирования - см. в числе прочих [6], [19], [10], [8], а также ссылки в этих работах.

Что же касается вычислений, то обычно опираются на численные методы решения стохастических дифференциальных уравнений и методы Монте-Карло - см. [5], [10], [9], [1]. Однако эти численные процедуры работают медленно и на практике предпочтительными могут оказаться аналитические формулы, позволяющие очень быстрые вычисления и не использующие какого-либо медленно работающего на компьютере численного метода.

Если говорить о точных аналитических аппроксимациях в контексте плавающей ренты, то, несмотря на их большую важность, результатов известно немного - см. [22] относительно Азиатских опционов и [21], [14] для плавающих рент специального типа. В данной работе мы предлагаем способ получения аналитических формул для плавающих рент в гораздо более общей модели. Наличие замкнутой аппроксимационной формулы весьма полезно не только с точки зрения прямого использования, но и для улучшения других численных методов: например, это может служить фундаментом для построения процедуры контроля уменьшения отклонений в симуляционных методах Монте-Карло (см. [13], а также недавнюю работу [4]).

Опишем базовый контракт с периодическими выплатами, обсуждаемый в данной работе. Такой контракт заключается между клиентом (страхователем) и страховщиком на конечное время $T>0$. Контракт зависит от

i) гарантированных платежей $\left(\delta_{i}\right)_{1 \leqslant i \leqslant n}$, которые выплачиваются страховщиком клиенту в некоторые фиксированные моменты времени $0<t_{1}<\cdots<t_{n} \leqslant T$ (мы полагаем также $t_{0}:=0, t_{n+1}:=T$ );

ii) взносов $\left(\alpha_{i}\right)_{1 \leqslant i \leqslant n}$, получаемых страховщиком в моменты $\left(t_{i}\right)_{1 \leqslant i \leqslant n}$ (взнос составляет некоторый процент от размера счета клиента, если этот счет положителен, и равен нулю в противном случае);

iii) динамики финансового индекса $S$, который описывает изменение размеров счета клиента между моментами $\left(t_{i}\right)_{0 \leqslant i \leqslant n+1}$ (данный индекс, как правило, соответствует капиталу, инвестированному в фондовый рынок и рынок облигаций, причем предполагается, что его волатильность ниже волатильности на фондовых рынках). 
Обозначим через $\left(\mathrm{A}_{t}^{\alpha, \delta}\right)_{t \geqslant 0}$ процесс изменения счета клиента с течением времени. Пусть $\mathrm{A}_{0}$ - объем средств, инвестированный клиентом в банковский счет в момент времени $t=0: \mathrm{A}_{0}^{\alpha, \delta}=\mathrm{A}_{0}$. Тогда эволюция счета определяется системой уравнений

$$
\begin{cases}\mathrm{A}_{t}^{\alpha, \delta}=\mathrm{A}_{t_{i}}^{\alpha, \delta} \frac{S_{t}}{S_{t_{i}}}, & t_{i} \leqslant t<t_{i+1}, 0 \leqslant i \leqslant n, \\ \mathrm{~A}_{t_{i}}^{\alpha, \delta}=\mathrm{A}_{t_{i}-}^{\alpha, \delta}-\alpha_{i}\left(\mathrm{~A}_{t_{i}-}^{\alpha, \delta}\right)_{+}-\delta_{i}, & 1 \leqslant i \leqslant n,\end{cases}
$$

где $x_{+}:=\max (x, 0)$. В момент окончания контракта клиент получает свой вклад в случае, если он положителен. Для оценивания страховщиком стоимости контракта с плавающей рентой используется методология совершенного хеджирования на полном рынке (см. [18] и библиографию в этой работе), предусматривающая вычисление риск-нейтрального ожидания дисконтированных выплат:

$$
\mathrm{VA}_{0}^{\alpha, \delta}=\mathbf{E}\left[D_{T}\left(\mathrm{~A}_{T}^{\alpha, \delta}\right)_{+}+\sum_{i=1}^{n} D_{t_{i}} \delta_{i}-\sum_{i=1}^{n} D_{t_{i}} \alpha_{i}\left(\mathrm{~A}_{t_{i}-}^{\alpha, \delta}\right)_{+}\right]
$$

где $D_{t}=\exp \left(-\int_{0}^{t} r_{s} d s\right)$ - дисконтирующий фактор, вычисленный на основе безрисковой процентной ставки $\left(r_{s}\right)$ и $\mathbf{E}[\cdot]$ - математическое ожидание относительно мартингальной меры Q. Даже в простейших моделях явный вид указанного математического ожидания неизвестен, и наша задача - найти какие-то простые и эффективные численные аппроксимации. На самом деле, для того чтобы контракт был справедливым, выплаты $\left(\delta_{i}\right)_{1 \leqslant i \leqslant n}$ должны быть организованы таким образом, чтобы $\mathrm{VA}_{0}^{\alpha, \delta}$ равнялась начальной инвестиции клиента $\mathrm{A}_{0}$ : это может быть достигнуто численно с использованием такой аналитической формулы для $\mathrm{VA}_{0}^{\alpha, \delta}$, как наша.

Заметим, что в расчетной формуле (2) мы пренебрегаем риском смертности. В случае, когда этот риск не зависит от рыночного риска, хорошо известно, что совершенное хеджирование для страховщика возможно, если число страхователей стремится к бесконечности и расчетная формула видоизменена дополнительным дисконтирующим фактором (см. работы Меллера [16], [17]). Следовательно, не составляет труда модифицировать наши аппроксимации с учетом риска смертности, и мы оставляем это читателю.

План работы. В разделе 2 мы описываем рассматриваемую модель (модель Блэка-Шоулса для $S$ и модель Халла-Уайта для $r$ ), а именно, мы показываем, как свести наш анализ $\mathrm{VA}_{0}^{\alpha, \delta}$ к более простой эквивалентной задаче, и аргументируем выбор смещенного логнормального аппроксиматора, который учитывает совместную асимптотику малой волатильности и малого платежа. В разделе 3 мы приводим основные аппроксимационные результаты (формулы и анализ ошибок) и даем 
планы доказательств. Полные доказательства отнесены в приложение (раздел 6). В разделе 4 представлены численные эксперименты, подтверждающие эффективность наших аппроксимаций.

\section{2. Модель и обозначения.}

2.1. Предположения. Мы предполагаем, что выплаты $\left(\delta_{i}\right)_{1 \leqslant i \leqslant n}$ и взносы $\left(\alpha_{i}\right)_{1 \leqslant i \leqslant n}$ являются детерминированными, как это обычно и бывает на практике; более того, пусть $\delta_{i} \geqslant 0$ и $\alpha_{i} \in[0,1]$. Для вероятностного моделирования относительно риск-нейтральной меры мы рассматриваем фильтрованное вероятностное пространство $\left(\Omega, \mathscr{F},\left(\mathscr{F}_{t}\right)_{t}, \mathbf{Q}\right)$ с обычными условиями на фильтрацию и предполагаем, что на этом пространстве определены два независимых винеровских процесса $W$ и $B$. Далее мы моделируем финансовый индекс $S$ с помощью геометрического броуновского движения с детерминированной волатильностью $\left(\sigma_{t}\right)_{t}$ :

$$
\frac{d S_{t}}{S_{t}}=r_{t} d t+\sigma_{t}\left(\rho d W_{t}+\sqrt{1-\rho^{2}} d B_{t}\right)
$$

Для простоты обозначений считаем, что $S_{0}=\mathrm{A}_{0}\left(=\mathrm{A}_{0}^{\alpha, \delta}\right)$. Поскольку плавающие ренты являются среднесрочными или долгосрочными контрактами, для целей риск-менеджмента необходимо учитывать также стохастические процентные ставки. Таким образом, мы выбираем однофакторную модель Халла-Уайта

$$
d r_{t}=\left(\theta_{t}-a r_{t}\right) d t+\sigma_{t}^{r} d W_{t}
$$

с детерминированными параметрами $\left(\theta_{t}, \sigma_{t}^{r}\right)_{t}$ и $a>0$. Нетрудно соотнести эту модель с гауссовскими многофакторными моделями. В этом случае параметр $\rho \in]-1,1[$ необходимо понимать как коэффициент корреляции между финансовым индексом (составленным из акций и облигаций) и процентными ставками. В нашей модели Халла-Уайта параметр $\theta$ подбирается таким образом, чтобы кривая доходности модели в начальный момент соответствовала рыночной, основанной на стоимости ${ }^{2}$ $\{B(0, t), t \geqslant 0\}$ облигаций без купонных платежей.

С одной стороны, модель (3), (4) является очень простой; с другой стороны, она учитывает стохастичность как финансового индекса, так и процентной ставки; кроме того, эта модель популярна у страховых компаний.

2.2. Упрощение задачи. Поскольку выплаты и взносы в (2) зависят только от неотрицательных значений процесса $\mathrm{A}^{\alpha, \delta}$, мы можем

2) Как обычно, $B(t, s)$ обозначает цену в момент времени $t$ облигации без купонных платежей с выплатой 1 евро в момент $s$. 
модифицировать динамику (1), рассмотрев новую модель (с теми же обозначениями):

$$
\begin{cases}\mathrm{A}_{t}^{\alpha, \delta}=\mathrm{A}_{t_{i}}^{\alpha, \delta} \frac{S_{t}}{S_{t_{i}}}, & t_{i} \leqslant t<t_{i+1}, 0 \leqslant i \leqslant n, \\ \mathrm{~A}_{t_{i}}^{\alpha, \delta}=\mathrm{A}_{t_{i}-}^{\alpha, \delta}-\alpha_{i} \mathrm{~A}_{t_{i}-}^{\alpha, \delta}-\delta_{i}, & 1 \leqslant i \leqslant n .\end{cases}
$$

Нетрудно проверить, что значение (2) при такой модификации процесса $\mathrm{A}^{\alpha, \delta}$ не меняется. Таким образом,

$$
\mathrm{VA}_{0}^{\alpha, \delta}=\mathbf{E}\left[D_{T}\left(\mathrm{~A}_{T}^{\alpha, \delta}\right)_{+}\right]+\sum_{i=1}^{n} \delta_{i} B\left(0, t_{i}\right)-\sum_{i=1}^{n} \alpha_{i} \mathbf{E}\left[D_{t_{i}}\left(\mathrm{~A}_{t_{i}-}^{\alpha, \delta}\right)_{+}\right] .
$$

Следовательно, остается вычислить математические ожидания

$$
\mathbf{E}\left[D_{T}\left(\mathrm{~A}_{T}^{\alpha, \delta}\right)_{+}\right], \quad \mathbf{E}\left[D_{t_{i}}\left(\mathrm{~A}_{t_{i}-}^{\alpha, \delta}\right)_{+}\right], \quad 1 \leqslant i \leqslant n,
$$

где $\mathrm{A}^{\alpha, \delta}$ имеет логнормальную динамику с аффинными скачками в моменты $t_{i}$. Такая динамика аналогична динамике акции с дискретной выплатой аффинных дивидендов. В отличие от [11], здесь процентные ставки являются стохастическими (важное свойство плавающей ренты), и для получения точных формул мы существенно используем преимущество «правильных» замен вероятностных мер.

Чтобы упростить изложение, мы уделяем основное внимание вычислению базового члена $\mathbf{E}\left[D_{T}\left(\mathrm{~A}_{T}^{\alpha, \delta}\right)_{+}\right]$, аналогичный анализ может быть затем применен к другим членам. Для удобства мы используем обозначение

$$
R_{t, T}=\frac{S_{T}}{S_{t}}
$$

2.3. Логнормальный аппроксиматор. По индукции в уравнении (5) мы находим следующее представление для $\mathrm{A}^{\alpha, \delta}$ (доказательство отнесено в приложение, см. п. 6.1).

Лемма 1. Положим $\pi_{j, i}=\left(1-\alpha_{j+1}\right) \cdots\left(1-\alpha_{i}\right)$ для $j \leqslant i$. Тогда

$$
\begin{aligned}
\mathrm{A}_{t_{i+1}-}^{\alpha, \delta} & =\pi_{0, i} S_{t_{i+1}}-\sum_{j=1}^{i} \delta_{j} \pi_{j, i} R_{t_{j}, t_{i+1}}, \quad 0 \leqslant i \leqslant n \\
\mathrm{~A}_{T}^{\alpha, \delta} & =\pi_{0, n} S_{T}-\sum_{j=1}^{n} \delta_{j} \pi_{j, n} R_{t_{j}, T} .
\end{aligned}
$$

В рамках нашей модели $\mathrm{A}_{T}^{\alpha, \delta}$ является суммой коррелированных логнормальных случайных величин и точное вычисление $\mathbf{E}\left[D_{T}\left(\mathrm{~A}_{T}^{\alpha, \delta}\right)_{+}\right]$невозможно. Обсудим вкратце идею выбираемой аппроксимации и перспективы нахождения количественных границ ошибки. В качестве аппроксимации можно было бы рассмотреть асимптотики малых платежей $\delta_{i} \rightarrow 0$, что привело бы к разложению в окрестности значения 
$\mathbf{E}\left[D_{T}\left(\pi_{0, n} S_{T}\right)_{+}\right] ;$в силу того, что дисконтированный процесс $S$ является положительным мартингалом, последнее математическое ожидание равно $\pi_{0, n} S_{0}$ независимо от величин выплат; очевидно, что подобный подход приводит к неаккуратным аппроксимациям. С другой стороны, можно было бы рассмотреть асимптотику с точки зрения малой волатильности $\left(\sigma_{t}, \sigma_{t}^{r} \rightarrow 0\right)$, но такой подход также неудовлетворителен, поскольку при этом член нулевого порядка является полностью детерминированным.

Наш подход сочетает обе упомянутье выще асимптотики $\left(\delta_{i}, \sigma_{t}, \sigma_{t}^{r} \rightarrow 0\right)$ следующим образом. Положим

$$
\widehat{\delta}_{i}=\delta_{i} \pi_{i, n}
$$

и определим аппроксиматор

$$
\bar{A}_{T}^{\alpha, \delta}=\pi_{0, n} S_{T}-\sum_{i=1}^{n} \widehat{\delta}_{i} \mathbf{E}\left[R_{t_{i}, T}\right]=: \pi_{0, n} S_{T}-K^{\alpha, \delta} .
$$

Это величина вклада с учетом среднего эффекта от выплат. В нашей модели $\mathbf{E}\left[R_{t_{i}, T}\right]$ вычисляется в явном виде (доказательство приводимой ниже леммы см. в п. 6.2 приложения).

Лемма 2. Пусть $\Gamma(t, u)=\int_{t}^{u} \sigma_{s}^{r} e^{-a(u-s)} d s$. При $0 \leqslant t \leqslant T$ uмеem место равенство

$$
\begin{aligned}
\mathbf{E}\left[R_{t, T}\right]=\frac{B(0, t)}{B(0, T)} \exp & \left(\int_{0}^{t}\left[\Gamma^{2}(s, T)-\Gamma(s, t) \Gamma(s, T)\right] d s\right. \\
& \left.+\int_{t}^{T}\left[\Gamma^{2}(s, T)-\rho \sigma_{s} \Gamma(s, T)\right] d s\right) .
\end{aligned}
$$

Значение $\mathbf{E}\left[D_{T}\left(\bar{A}_{T}^{\alpha, \delta}\right)_{+}\right]$является главным членом в аппроксимации $\mathbf{E}\left[D_{T}\left(\mathrm{~A}_{T}^{\alpha, \delta}\right)_{+}\right]$; некоторые корректирующие члены будут вычислены в следующем разделе. Приведенные ниже факторы позволяют думать, что эта аппроксимация нулевого порядка точна:

1) выплаты малы, что уменьшает влияние замены $R_{t_{i}, T}$ другой величиной;

2 ) волатильности $r$ и $S$ малы, поэтому $R_{t_{i}, T}$ близко к своему математическому ожиданию.

По поводу количественного описания и строгих оценок ошибок см. теорему 5 .

Наконец, заметим, что $\bar{A}_{T}^{\alpha, \delta}$ - смещенная логнормальная случайная величина (так называемый логнормальный аппроксиматор); таким образом, $\mathbf{E}\left[D_{T}\left(\bar{A}_{T}^{\alpha, \delta}\right)_{+}\right]$снова может быть вычислено в явном виде за счет использования смещенной цены исполнения $K^{\alpha, \delta}$ в формуле Блэка (см., например, [18]). 
Предложение 1. Положим $K^{\alpha, \delta}:=\sum_{i=1}^{n} \widehat{\delta}_{i} \mathbf{E}\left[R_{t_{i}, T}\right]$. Тогда

$$
\mathbf{E}\left[D_{T}\left(\bar{A}_{T}^{\alpha, \delta}\right)_{+}\right]=B(0, T) \mathscr{C}\left(\frac{\pi_{0, n}}{B(0, T)} S_{0}, K^{\alpha, \delta}\right),
$$

где

$$
\begin{aligned}
\mathscr{C}(x, k) & =x \mathscr{N}\left[d_{+}(x, k)\right]-k \mathscr{N}\left[d_{-}(x, k)\right] \\
d_{ \pm}(x, k) & =\frac{1}{\Sigma \sqrt{T}} \ln \frac{x}{k} \pm \frac{1}{2} \Sigma \sqrt{T} \\
\Sigma^{2} & =\frac{1}{T} \int_{0}^{T}\left(\Gamma^{2}(s, T)+2 \rho \sigma_{s} \Gamma(s, T)+\sigma_{s}^{2}\right) d s
\end{aligned}
$$

а⿻上丨 - функиия распределения стандартной нормальной случайной величиньл.

Заметим, что $K^{\alpha, \delta}$ может быть получена в явном виде в силу леммы 2 и уравнения (7).

Вышеупомянутая величина является главным членом в разложении $\mathbf{E}\left[D_{T}\left(\mathrm{~A}_{T}^{\alpha, \delta}\right)_{+}\right]$; в следующем разделе нашей целью будет, во-первых, нахождение корректирующих членов, что позволит нам достичь большей точности и, во-вторых, вывод оценок для ошибок в соответствии с параметрами модели. Следующие утверждения будут играть ключевую роль в выводе явных формул. Эти утверждения тесно связаны с гауссовской моделью (3), (4), доказательство утверждения 2 отнесено в приложение (см. п. 6.3).

Предложение 2. Для $k \in \mathbf{N}^{*} u\left(i_{1}, \cdots, i_{k}\right) \in\{0,1, \ldots, n+1\}^{k}$ nоложим

$$
c_{i_{1}, \cdots, i_{k}}:=\mathbf{E}\left[D_{T} R_{t_{i_{1}}, T} R_{t_{i_{2}}, T} \cdots R_{t_{i_{k}}, T}\right] .
$$

Если $i_{1} \leqslant \cdots \leqslant i_{k}$, то, используя условия $t_{i_{0}}:=0 u t_{i_{k+1}}:=T$, мьь имеем

$$
\begin{aligned}
c_{i_{1}, i_{2}, \ldots, i_{k}}= & \frac{\prod_{j=1}^{k} B\left(0, t_{i_{j}}\right)}{B(0, T)^{k-1}} \\
\times \exp \left(\frac{1}{2} \sum_{j=0}^{k} \int_{t_{i_{j}}}^{t_{i_{j+1}}}[\right. & (k-1) \Gamma^{2}(s, T)-j \sigma_{s}^{2}-\sum_{l=j+1}^{k} \Gamma^{2}\left(s, t_{i_{l}}\right) \\
& +\left[(k-1) \Gamma(s, T)+j \rho \sigma_{s}-\sum_{l=j+1}^{k} \Gamma\left(s, t_{i_{l}}\right)\right]^{2} \\
& \left.\left.+j^{2}\left(1-\rho^{2}\right) \sigma_{s}^{2}\right] d s\right) .
\end{aligned}
$$

$B$ случае неупорядоченньх индексов $\left(i_{1}, \ldots, i_{k}\right)$ та же самая формула справедлива после упорядочения индексов по возрастанию.

$B$ частности, $c_{i}=B\left(0, t_{i}\right)$ u $c_{0, n+1}=1$. 
Если речь идет о замене меры с логнормальной плотностью РадонаНикодима $L$ и если $(L, M)$ - логнормальный вектор, хорошо известно, что $M$ остается распределенной логнормально (относительно новой меры) и только ее среднее меняется. Мы заостряем внимание на полезных заменах меры.

Предложение 3. В обозначениях утверждения 2 определим вероятностную меру ${ }^{3)} \mathbf{Q}_{t_{i_{1}}, \ldots, t_{i_{k}}}$ плотностью

$$
\left.\frac{d \mathbf{Q}_{t_{i_{1}}, \ldots, t_{i_{k}}}}{d \mathbf{Q}}\right|_{\mathscr{F}_{T}}:=\frac{D_{T} R_{t_{i_{1}}, T} \cdots R_{t_{i_{k}}, T}}{c_{i_{1}, \ldots, i_{k}}}=L_{t_{i_{1}}, \ldots, t_{i_{k}}} .
$$

Тогда относительно $\mathbf{Q}_{t_{i_{1}}, \ldots, t_{i_{k}}}$ аппроксиматор $\bar{A}_{T}^{\alpha, \delta}$ имеет то же распределение, что смещенная логнормальная случайная величина

$$
\pi_{0, n} \frac{c_{0, i_{1}, \ldots, i_{k}}}{c_{i_{1}, \ldots, i_{k}}} \widetilde{S}_{T}-K^{\alpha, \delta}
$$

относительно Q, где $\ln \widetilde{S}_{T} \stackrel{\text { Q-law }}{=} \mathscr{N}\left(\ln S_{0}-(1 / 2) \Sigma^{2} T, \Sigma^{2} T\right)$.

Д о к а з а т е л ь с т в о. Напомним (см. (8)), что $\bar{A}_{T}^{\alpha, \delta}=\pi_{0, n} S_{T}-$ $K^{\alpha, \delta}$. Тогда $S_{T}$ относительно $\mathbf{Q}_{t_{i_{1}}, \ldots, t_{i_{k}}}$ является логнормальной величиной с той же совокупной дисперсией $\Sigma^{2} T$. Остается вычислить аппроксимацию среднего:

$$
\mathbf{E}_{\mathbf{Q}_{t_{i_{1}}, \ldots, t_{i_{k}}}}\left[S_{T}\right]=\frac{\mathbf{E}\left[S_{T} D_{T} R_{t_{i_{1}}, T} \cdots R_{t_{i_{k}}, T}\right]}{c_{i_{1}, \ldots, i_{k}}}=S_{0} \frac{c_{0, i_{1}, \ldots, i_{k}}}{c_{i_{1}, \ldots, i_{k}}} .
$$

Это приводит к заявленному результату.

3. Основные результаты. Теперь мы готовы сформулировать наши основные аппроксимационные результаты для $\mathbf{E}\left[D_{T} h\left(\mathrm{~A}_{T}^{\alpha, \delta}\right)\right]$, где $h(x)=x_{+}$. Наша методология основывается на разложении Тейлора и искусных вычислениях корректирующих членов с использованием аппроксиматора $\bar{A}_{T}^{\alpha, \delta}:$ в силу леммы 1 и уравнения (8) расстояние от аппроксиматора имеет простое представление

$$
\mathrm{A}_{T}^{\alpha, \delta}-\bar{A}_{T}^{\alpha, \delta}=-\sum_{i=1}^{n} \widehat{\delta}_{i}\left(R_{t_{i}, T}-\mathbf{E}\left[R_{t_{i}, T}\right]\right)
$$

Сначала мы рассматриваем гладкие функции выплат $h$; переход к случаю $h(x)=x_{+}$можно осуществить с помощью методов работы [11].

3) Если $t_{i_{1}}=T$ (т.е. $\left.i_{1}=n+1\right)$, то $\mathbf{Q}_{t_{i_{1}}}$ является обычной $T$-форвардной мерой; если же $t_{i_{1}}=0$ (т.е. $i_{1}=0$ ), то $\mathbf{Q}_{t_{i_{1}}}$ - мера, соответствующая индексу $S$ (см. $[18$, гл. 8]. 


\section{1. Аппроксимационные формулы в случае гладкой функ- ции $h$.}

О п р е д е л е н и е 1 . Мы говорим, что функция $h: \mathbf{R} \mapsto \mathbf{R}$ удовлетворяет условию $\mathscr{C}_{\text {pol }}^{k}$, если она $k$ раз непрерывно дифференцируема и ее производные растут полиномиально: для некоторых положительных постоянных $C_{h}$ и $p_{h}$ выполнено неравенство $\sum_{j=0}^{k}\left|h^{(j)}(x)\right| \leqslant C_{h}\left(1+|x|^{p_{h}}\right)$ при всех $x \in \mathbf{R}$.

Теорема 1. Пусть функция $h$ удовлетворяет условию $\mathscr{C}_{\mathrm{pol}}^{2}$. Тогда

$$
\begin{aligned}
\mathbf{E}\left[D_{T} h\left(\mathrm{~A}_{T}^{\alpha, \delta}\right)\right]= & B(0, T) \mathbf{E}\left[h\left(\frac{\pi_{0, n}}{B(0, T)} \widetilde{S}_{T}-K^{\alpha, \delta}\right)\right] \\
& +\sum_{i=1}^{n} \widehat{\delta}_{i} c_{i} \partial_{K} \mathbf{E}\left[h\left(\pi_{0, n} \frac{c_{0, i}}{c_{i}} \widetilde{S}_{T}-K^{\alpha, \delta}\right)\right] \\
& -K^{\alpha, \delta} B(0, T) \partial_{K} \mathbf{E}\left[h\left(\frac{\pi_{0, n}}{B(0, T)} \widetilde{S}_{T}-K^{\alpha, \delta}\right)\right]+\operatorname{Error}_{1}(h),
\end{aligned}
$$

где ошибка $\operatorname{Error}_{1}(h)$ оченивается в теореме 3. Здесь производная

$$
\partial_{K} \mathbf{E}\left[h\left(\frac{\pi_{0, n}}{B(0, T)} \widetilde{S}_{T}-K^{\alpha, \delta}\right)\right]
$$

ecmb

$$
\left.\partial_{K} \mathbf{E}\left[h\left(\frac{\pi_{0, n}}{B(0, T)} \widetilde{S}_{T}-K\right)\right]\right|_{K=K^{\alpha, \delta}} .
$$

В правой части равенства, определяющего разложение, функция $h$ вычисляется на различных смещенных логнормальных случайных величинах: это позволяет осуществить простые численные расчеты (точные в случае функции $\left.h: x \mapsto x_{+}\right)$. Заметим, что $\mathbf{E}\left[R_{t_{i}, T}\right], K^{\alpha, \delta}, c_{i}$ и $c_{0, i}$ известны в явном виде в силу леммы 2 и утверждения 2 .

Сейчас мы докажем теорему 1 показав, как замены меры в утверждении 3 позволяют представить корректирующие члены. Доказательства более точных аппроксимаций отнесены в приложение (раздел 6).

Д о к а з а т е л ь с т в о. Используя разложение Тейлора до первого порядка и представление (9), находим, что

$$
\begin{aligned}
\mathbf{E}\left[D_{T} h\left(\mathrm{~A}_{T}^{\alpha, \delta}\right)\right]= & \mathbf{E}\left[D_{T} h\left(\bar{A}_{T}^{\alpha, \delta}\right)\right]-\sum_{i=1}^{n} \widehat{\delta}_{i} \mathbf{E}\left[D_{T}\left(R_{t_{i}, T}-\mathbf{E}\left[R_{t_{i}, T}\right]\right) h^{\prime}\left(\bar{A}_{T}^{\alpha, \delta}\right)\right] \\
& +\operatorname{Error}_{1}(h) .
\end{aligned}
$$

Применяя утверждения 3 и 2 с мерой $\mathbf{Q}_{T}$, получаем

$$
\begin{aligned}
\mathbf{E}\left[D_{T} h\left(\bar{A}_{T}^{\alpha, \delta}\right)\right] & =B(0, T) \mathbf{E}_{\mathbf{Q}_{T}}\left[h\left(\bar{A}_{T}^{\alpha, \delta}\right)\right] \\
& =B(0, T) \mathbf{E}\left[h\left(\pi_{0, n} \frac{\widetilde{S}_{T}}{B(0, T)}-K^{\alpha, \delta}\right)\right] .
\end{aligned}
$$


Учитывая второй член в правой части (10), мы приходим к следующему равенству: для всех $i$

$$
\begin{aligned}
& \mathbf{E}\left[D_{T}\left(R_{t_{i}, T}-\mathbf{E}\left[R_{t_{i}, T}\right]\right) h^{\prime}\left(\bar{A}_{T}^{\alpha, \delta}\right)\right] \\
& =\mathbf{E}\left[D_{T} R_{t_{i}, T} h^{\prime}\left(\bar{A}_{T}^{\alpha, \delta}\right)\right]-\mathbf{E}\left[R_{t_{i}, T}\right] \mathbf{E}\left[D_{T} h^{\prime}\left(\bar{A}_{T}^{\alpha, \delta}\right)\right] .
\end{aligned}
$$

Величина $\mathbf{E}\left[D_{T} h^{\prime}\left(\bar{A}_{T}^{\alpha, \delta}\right)\right]$ в правой части может быть записана как производная (условие на $h$ позволяет менять местами дифференцирование и математическое ожидание):

$$
\mathbf{E}\left[D_{T} h^{\prime}\left(\bar{A}_{T}^{\alpha, \delta}\right)\right]=-\left.\partial_{K} \mathbf{E}\left[D_{T} h\left(\pi_{0, n} S_{T}-K\right)\right]\right|_{K=K^{\alpha, \delta}} .
$$

Это представление важно с точки зрения интерпретации в дальнейшем членов разложения как производных по параметрам модели (отсюда, потенциально представимых в явном виде).

Используя утверждения 3 и 2, мы преобразуем первое слагаемое в правой части $(11)$, интерпретируя множитель $D_{T} R_{t_{i}, T}$ (с точностью до нормирующей константы) как замену меры:

$$
\begin{aligned}
\mathbf{E}\left[D_{T} R_{t_{i}, T} h^{\prime}\left(\bar{A}_{T}^{\alpha, \delta}\right)\right] & =c_{i} \mathbf{E}_{\mathbf{Q}_{t_{i}}}\left[h^{\prime}\left(\bar{A}_{T}^{\alpha, \delta}\right)\right]=c_{i} \mathbf{E}\left[h^{\prime}\left(\pi_{0, n} \frac{c_{0, i}}{c_{i}} \widetilde{S}_{T}-K^{\alpha, \delta}\right)\right] \\
& =-\left.c_{i} \partial_{K} \mathbf{E}\left[h\left(\pi_{0, n} \frac{c_{0, i}}{c_{i}} \widetilde{S}_{T}-K\right)\right]\right|_{K=K^{\alpha, \delta}} .
\end{aligned}
$$

Объединяя это равенство с (11), (12) и подставляя в (10), мы получаем требуемый результат. Теорема 1 доказана.

Аналогичным образом мы находим разложение более высокого порядка (подробное доказательство см. в п. 6.4).

Теорема 2. Пусть функиия $h$ гладкая и удовлетворяет условию $\mathscr{C}_{\text {pol }}^{3}$. Тогда

$$
\begin{aligned}
& \mathbf{E}\left[D_{T} h\left(\mathrm{~A}_{T}^{\alpha, \delta}\right)\right]=B(0, T) \mathbf{E}\left[h\left(\frac{\pi_{0, n}}{B(0, T)} \widetilde{S}_{T}-K^{\alpha, \delta}\right)\right] \\
& +\sum_{i=1}^{n} \widehat{\delta}_{i} c_{i} \partial_{K} \mathbf{E}\left[h\left(\pi_{0, n} \frac{c_{0, i}}{c_{i}} \widetilde{S}_{T}-K^{\alpha, \delta}\right)\right] \\
& -K^{\alpha, \delta} B(0, T) \partial_{K} \mathbf{E}\left[h\left(\frac{\pi_{0, n}}{B(0, T)} \widetilde{S}_{T}-K^{\alpha, \delta}\right)\right] \\
& +\frac{1}{2}\left\{\sum_{i, j=1}^{n} \widehat{\delta}_{i} \widehat{\delta}_{j} c_{i, j} \partial_{K}^{2} \mathbf{E}\left[h\left(\pi_{0, n} \frac{c_{0, i, j}}{c_{i, j}} \widetilde{S}_{T}-K^{\alpha, \delta}\right)\right]\right. \\
& \quad-2 K^{\alpha, \delta} \sum_{i=1}^{n} \widehat{\delta}_{i} c_{i} \partial_{K}^{2} \mathbf{E}\left[h\left(\pi_{0, n} \frac{c_{0, i}}{c_{i}} \widetilde{S}_{T}-K^{\alpha, \delta}\right)\right] \\
& \left.+\left(K^{\alpha, \delta}\right)^{2} B(0, T) \partial_{K}^{2} \mathbf{E}\left[h\left(\frac{\pi_{0, n}}{B(0, T)} \widetilde{S}_{T}-K^{\alpha, \delta}\right)\right]\right\}+ \text { Error }_{2}(h),
\end{aligned}
$$

оценка для $\operatorname{Error}_{2}(h)$ приводится в теореме 3. 
Наконец, мы формулируем результаты относительно оценок ошибки аппроксимации. Доказательство отнесено в приложение (см. п. 6.5).

Теорема 3. Пусть функиия $h$ удовлетворяет условию $\mathscr{C}_{\mathrm{pol}}^{k+1}(k=1$ или $k=2)$ с некоторыми константами $C_{h} u p_{h}$. Тогда справедлива оченка

$$
\begin{aligned}
& \left|\operatorname{Error}_{k}(h)\right| \leqslant c C_{h}\left(1+S_{0}^{p_{h}}\right) \\
& \quad \times\left[n \sup _{1 \leqslant i \leqslant n}\left(\widehat{\delta}_{i}\left[\sup _{0 \leqslant t \leqslant T}\left|\sigma_{t}^{r}\right| \sqrt{T} \min \left(T, a^{-1}\right)+\sup _{0 \leqslant t \leqslant T}\left|\sigma_{t}\right| \sqrt{T-t_{i}}\right]\right)\right]^{k+1},
\end{aligned}
$$

где с есть константа (не зависящая от $h$ ), которая остается равномерно ограниченной, когда параметры модели стремятся $\kappa 0$.

Ввиду наличия в формуле теоремы $3(k+1)$-й степени, $k$ дает порядок аппроксимационной формулы: первый порядок для теоремы 1 и второй порядок для теоремы 2. В соответствии с выбираемым аппроксиматором (см. обсуждение после леммы 2), параметры точности есть комбинация платежей $\left(\delta_{i}\right)_{i}$ и волатильностей $\left(\sigma^{r}, \sigma\right)$; поэтому следует ожидать, что малые значения этих параметров увеличивают точность. С другой стороны, большие сроки контрактов $T$ могут ухудшить нашу аппроксимацию.

Неасимптотические оценки ошибок, приведенные в теореме 3 , должны рассматриваться как ориентир для оценки применимости и точности аппроксимационных формул. Интересным вариантом использования является взятие наших аппроксимаций в качестве контрольных в случае, когда платежи становятся зависимыми между собой. Даже когда параметры не являются малыми, представленные формулы могут быть использованы для эффективных урезаний погрешности, как сделано в работах [2], [4].

\section{2. Аппроксимационные формулы для положительной ча-} сти функции. Теоремы 1-3 можно распространить на случай $h(x)=$ $x_{+}$, аппроксимируя последовательностью гладких функций $\left(h_{m}\right)_{m}$ таких, что $\left|h_{m}^{\prime}(x)\right| \leqslant 1$, и переходя затем к пределу; подобная техника успешно использовалась в [11], где применение многих технических элементов исчисления Малявэна помогло доказать, что зависимость оценок ошибки от $h$ может быть сведена к зависимости от $h_{m}^{\prime}$ (равномерно ограниченной). Мы опускаем анализ ошибок и приводим только аппроксимационные формулы.

Теорема 4. Разложение математического ожидания $\mathbf{E}\left[D_{T}\left(\mathrm{~A}_{T}^{\alpha, \delta}\right)_{+}\right]$ до первого порядка имеет вид

$$
\mathbf{E}\left[D_{T}\left(\mathrm{~A}_{T}^{\alpha, \delta}\right)_{+}\right]=B(0, T) \mathscr{C}\left(\frac{\pi_{0, n}}{B(0, T)} S_{0}, K^{\alpha, \delta}\right)
$$




$$
\begin{aligned}
& +\sum_{i=1}^{n} \widehat{\delta}_{i} c_{i} \partial_{K} \mathscr{C}\left(\frac{\pi_{0, n} c_{0, i}}{c_{i}} S_{0}, K^{\alpha, \delta}\right) \\
& -K^{\alpha, \delta} B(0, T) \partial_{K} \mathscr{C}\left(\frac{\pi_{0, n}}{B(0, T)} S_{0}, K^{\alpha, \delta}\right)+\operatorname{Error}_{1}\left((\cdot)_{+}\right)
\end{aligned}
$$

Разложение до второго порядка имеет вид

$\mathbf{E}\left[D_{T}\left(\mathrm{~A}_{T}^{\alpha, \delta}\right)_{+}\right]=$членьи первого порядка

$$
\begin{aligned}
& +\frac{1}{2}\left\{\sum_{i, j=1}^{n} \widehat{\delta}_{i} \widehat{\delta}_{j} c_{i, j} \partial_{K}^{2} \mathscr{C}\left(\frac{\pi_{0, n} c_{0, i, j}}{c_{i, j}} S_{0}, K^{\alpha, \delta}\right)\right. \\
& -2 K^{\alpha, \delta} \sum_{i=1}^{n} \widehat{\delta}_{i} c_{i} \partial_{K}^{2} \mathscr{C}\left(\frac{\pi_{0, n} c_{0, i}}{c_{i}} S_{0}, K^{\alpha, \delta}\right) \\
& \left.+\left(K^{\alpha, \delta}\right)^{2} B(0, T) \partial_{K}^{2} \mathscr{C}\left(\frac{\pi_{0, n}}{B(0, T)} S_{0}, K^{\alpha, \delta}\right)\right\}+ \text { Error }_{2}\left((\cdot)_{+}\right) .
\end{aligned}
$$

Важно отметить, что эти результаты могут быть распространены на произвольный порядок $k$. В этом случае вычисление $k$-го члена аппроксимационной формулы сводится к вычислению величин

$$
\mathbf{E}\left[D_{T} R_{t_{i_{1}}, T} R_{t_{i_{2}}, T} \cdots R_{t_{i_{k}}, T} h^{(k)}\left(\bar{A}_{T}^{\alpha, \delta}\right)\right] .
$$

Используя утверждение 3 с вероятностной мерой $\mathbf{Q}_{t_{i_{1}}, \ldots, t_{i_{k}}}$ и вычисления, аналогичные предыдущим, можно получить, что эта величина есть, с точностью до множителя явного вида, $k$-я производная формулы Блэка по цене исполнения:

$$
\begin{aligned}
& \mathbf{E}\left[D_{T} R_{t_{i_{1}}, T} R_{t_{i_{2}}, T} \ldots R_{t_{i_{k}}, T} h^{(k)}\left(\bar{A}_{T}^{\alpha, \delta}\right)\right] \\
& =(-1)^{k} c_{i_{1}, \ldots, i_{k}} \partial_{K}^{k} \mathbf{E}\left[h\left(\pi_{0, n} \frac{c_{0, i_{1}, \ldots, i_{k}}}{c_{i_{1}, \ldots, i_{k}}} \widetilde{S}_{T}-K^{\alpha, \delta}\right)\right] .
\end{aligned}
$$

Для $k=3$ аппроксимация записывается следующим образом.

Теорема 5. Разложение до третьего порядка имеет вид $\mathbf{E}\left[D_{T}\left(\mathrm{~A}_{T}^{\alpha, \delta}\right)_{+}\right]=$членьв второго порядка

$$
\begin{aligned}
& +\frac{1}{6}\left\{\sum_{i, j, l=1}^{n} \widehat{\delta}_{i} \widehat{\delta}_{j} \widehat{\delta}_{l} c_{i, j, l} \partial_{K}^{3} \mathscr{C}\left(\frac{\pi_{0, n} c_{0, i, j, l}}{c_{i, j, l}} S_{0}, K^{\alpha, \delta}\right)\right. \\
& -3 K^{\alpha, \delta} \sum_{i, j=1}^{n} \widehat{\delta}_{i} \widehat{\delta}_{j} c_{i, j} \partial_{K}^{3} \mathscr{C}\left(\frac{\pi_{0, n} c_{0, i, j}}{c_{i, j}} S_{0}, K^{\alpha, \delta}\right) \\
& +3\left(K^{\alpha, \delta}\right)^{2} \sum_{i=1}^{n} \widehat{\delta}_{i} c_{i} \partial_{K}^{3} \mathscr{C}\left(\frac{\pi_{0, n} c_{0, i}}{c_{i}} S_{0}, K^{\alpha, \delta}\right) \\
& \left.-\left(K^{\alpha, \delta}\right)^{3} B(0, T) \partial_{K}^{3} \mathscr{C}\left(\frac{\pi_{0, n}}{B(0, T)} S_{0}, K^{\alpha, \delta}\right)\right\}+\operatorname{Error}_{3}\left((\cdot)_{+}\right) .
\end{aligned}
$$


Стоит отметить, что оценки ошибки $\operatorname{Error}_{k}(h)$, приведенные в теореме 3 , можно, используя метод работ [3], [11], распространить на случай $h(x)=x_{+}$при условии, что волатильность $\sigma_{t}$ не стремится к нулю.

Тот факт, что границы для ошибок увеличиваются в ростом $n$ и $T$, может смутить: действительно, это создает трудности в случае $h(x)=$ $x_{+}$, поскольку цена исполнения $K^{\alpha, \delta}$ также увеличивается с ростом $n$ и $T$, что делает опцион все более и более невыгодным (цена и производные по параметрам стремятся к 0). Дальнейшие эксперименты подтверждают наблюдение, что точность не теряется с ростом $n$ и $T$.

4. Численные эксперименты. В этом разделе мы обсуждаем с численной точки зрения точность формул в зависимости от параметров модели и порядка $k$ аппроксимационных формул (обозначение: $\mathrm{AF}_{k}$ ). Тестовое значение определяется с помощью метода Монте-Карло, выполненного с $10^{8}$ симуляций (мы предлагаем симметричные 95\% доверительные интервалы). Контракт с плавающей рентой предусматривает, что платежи $\delta_{i}$ (взносы $\alpha_{i}$ ) получаются (платятся) ежегодно в течение периода $T=20$ лет (взносы и платежи осуществляются также и в год $T$ ). Взносы постоянны: $\alpha_{i}=0.9 \%$. Платежи начинаются с шестого года: $\delta_{i}=0$ для $i \leqslant 5$ и $\delta_{i}=\delta$ для $i \geqslant 6$ (где $\delta$ определяется в последующих тестах). Параметры модели равны $S_{0}=100, \rho=0$, а значения $(B(0, t): 0 \leqslant t \leqslant T)$ указаны в таблице 1 . Мы считаем, что параметры $\delta_{t}$ и $\delta_{t}^{r}$ не зависят от времени, так что коэффициенты в лемме 2 , утверждении 2, теоремах 4 и 5 могут быть сразу вычислены без дополнительного численного интегрирования (смотрите [2] об упрощенных формулах в этой форме).

Таблица 1. Начальная стоимость облигации без купонных платежей

\begin{tabular}{|c|c|c|c|c|c|c|c|c|c|c|c|c|c|}
\hline$t$ & \multicolumn{2}{|c|}{1} & \multicolumn{2}{|c|}{2} & \multicolumn{2}{|c|}{3} & \multicolumn{2}{|c|}{4} & \multicolumn{2}{|c|}{5} & \multicolumn{2}{|c|}{6} & 7 \\
\hline$B(0, t)$ & \multicolumn{2}{|c|}{0.98644} & \multicolumn{2}{|c|}{0.96937} & \multicolumn{2}{|c|}{0.94772} & \multicolumn{2}{|c|}{0.92097} & \multicolumn{2}{|c|}{0.89055} & \multicolumn{2}{|c|}{0.85861} & 0.82600 \\
\hline$t$ & \multicolumn{2}{|c|}{8} & \multicolumn{2}{|c|}{9} & \multicolumn{2}{|c|}{10} & \multicolumn{2}{|c|}{11} & \multicolumn{2}{|c|}{12} & \multicolumn{2}{|c|}{13} & 14 \\
\hline$B(0, t)$ & \multicolumn{2}{|c|}{0.79357} & \multicolumn{2}{|c|}{0.76185} & \multicolumn{2}{|c|}{0.73081} & \multicolumn{2}{|c|}{0.70019} & \multicolumn{2}{|c|}{0.67039} & \multicolumn{2}{|c|}{0.64196} & 0.61517 \\
\hline & $t$ & & 5 & & & & & & 8 & & 9 & & \\
\hline \multicolumn{2}{|c|}{$B(0, t)$} & \multicolumn{2}{|c|}{0.59018} & \multicolumn{2}{|c|}{0.56706} & \multicolumn{2}{|c|}{0.54581} & \multicolumn{2}{|c|}{0.52635} & \multicolumn{2}{|c|}{0.50858} & \multicolumn{2}{|c|}{0.49235} \\
\hline
\end{tabular}

Таблица 2. Оценки величины $\mathrm{VA}_{0}^{\alpha, \delta}$, полученные различными методами (для $\sigma=10 \%$ и $\delta=9.5)$.

\begin{tabular}{|c|c|c|c|c|c|c|c|}
\hline$\sigma^{r}$ & $a$ & $\mathrm{MC}$ & $\mathrm{AF}_{1}$ & $\mathrm{AF}_{2}$ & $\mathrm{AF}_{3}$ & $\mathrm{AF}_{4}$ & $\mathrm{AF}_{5}$ \\
\hline 0.01 & 0.001 & $94.7514 \pm 0.0038$ & 93.5575 & 94.2223 & 94.5405 & 94.6740 & 94.7220 \\
0.01 & 0.041 & $93.8524 \pm 0.0037$ & 92.6340 & 93.3830 & 93.7057 & 93.8169 & 93.8434 \\
0.01 & 0.091 & $93.1718 \pm 0.0036$ & 91.9120 & 92.7376 & 93.0658 & 93.1593 & 93.1704 \\
\hline 0.02 & 0.001 & $100.673 \pm 0.004$ & 98.9330 & 99.6428 & 100.056 & 100.296 & 100.437 \\
0.02 & 0.041 & $98.3116 \pm 0.0045$ & 96.9445 & 97.6249 & 97.9742 & 98.1439 & 98.2236 \\
0.02 & 0.091 & $96.3348 \pm 0.0042$ & 95.0395 & 95.7890 & 96.1272 & 96.2593 & 96.3035 \\
\hline
\end{tabular}



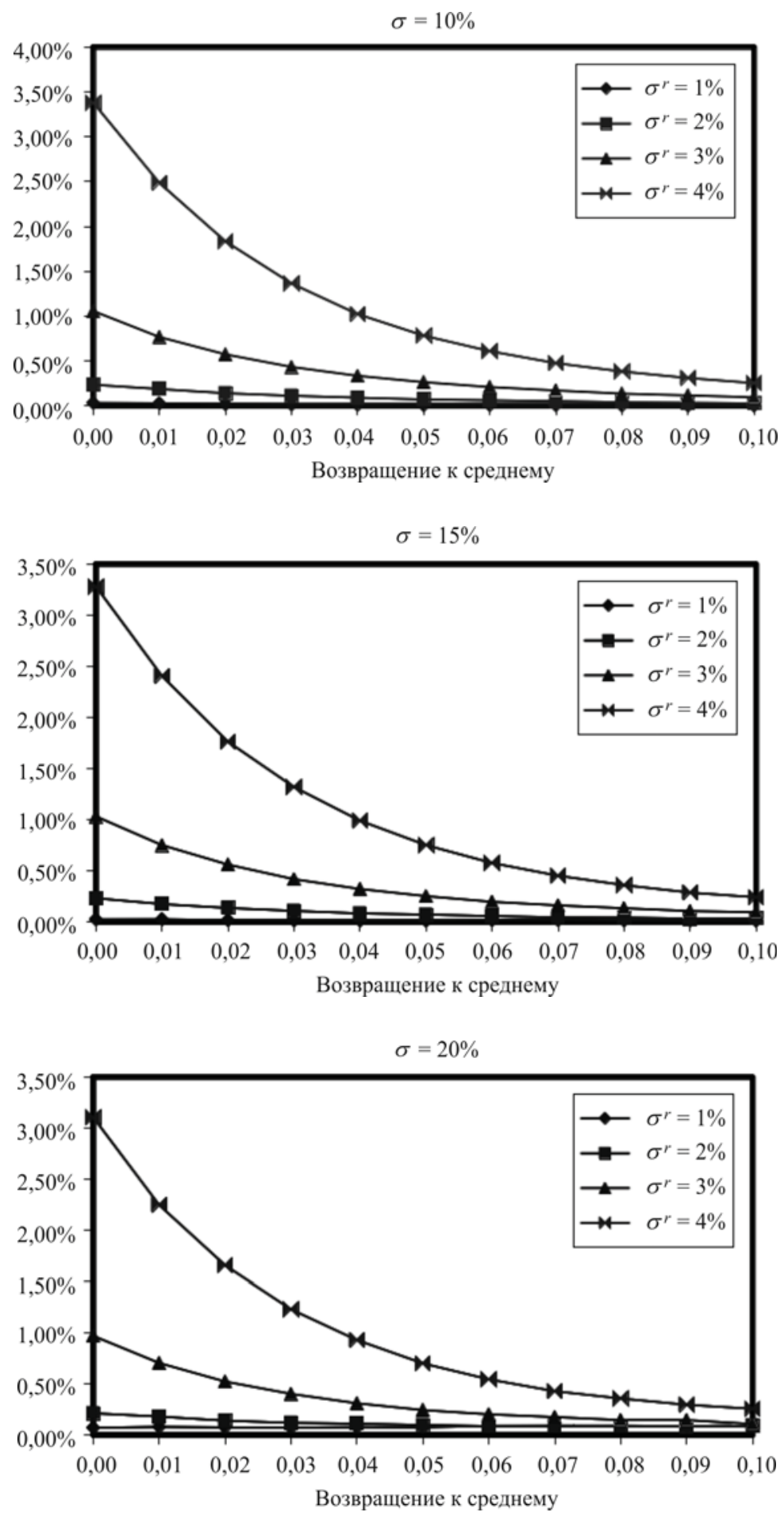

Рис. 1. Относительная ошибка оценки $\mathrm{AF}_{5}$ как функция среднего $a$ для различных значений волатильности $\sigma^{r}$ процентной ставки и волатильности $\sigma$ при $\delta=9.5$. 
Для различных значений волатильности $\sigma^{r}$ процентной ставки и возвращающего среднего $a$ в таблице 2 указаны тестовое значение (столбец MC) и значения, вычисленные на основе аппроксимационных формул порядка $k=1, \ldots, 5$ (столбцы $\mathrm{AF}_{1}, \ldots, \mathrm{AF}_{5}$ соответственно). Как и ожидалось, чем выше порядок, тем лучше точность. Таким образом, значение $\mathrm{AF}_{5}$ является очень точным для всех значений $\sigma^{r}$ и $a$. Однако и более низких порядков иногда достаточно для получения хорошей точности. Кроме того, по сравнению с методами Монте-Карло велик выигрыш во времени вычислений: нахождение $\mathrm{AF}_{5}$ занимает около 2 секунд, в то время как для вычисления с той же точностью симуляциями Монте-Карло требуется около 2 часов.

На рис. 1 показана относительная ошибка для $\mathrm{AF}_{5}$ как функция $a$ и $\sigma^{r}$ для трех значений $\sigma(10 \%, 15 \%, 20 \%)$. Стоит отметить, что $a$ и $\sigma^{r}$ играют существенную роль, особенно если $a$ меньше $2 \%$; действительно, относительная ошибка сильно увеличивается, когда $а$ уменьшается или $\sigma^{r}$ возрастает. Это согласуется с оценкой ошибки в теореме 3 (для случая $k=5$ ), которая пропорциональна $\sigma^{r}$ и $a^{-1}$. С другой стороны, волатильность индекса $S$ оказывает меньшее влияние. Это соответствует тому факту, что в теореме $3 \sigma^{r}$-фактор есть $T \sqrt{T}$, в то время как $\sigma$ фактор $-\sqrt{T}$.

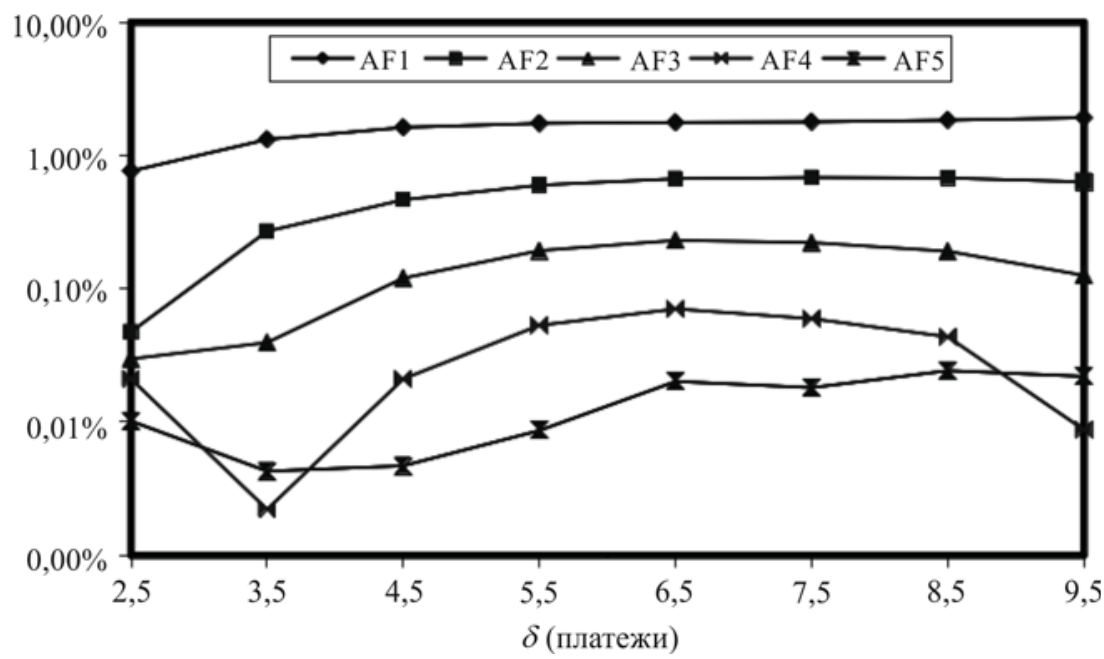

Рис. 2. Относительная ошибка (по логарифмической шкале) $\mathrm{AF}_{k}(k=1, \ldots, 5)$ как функция ежегодных платежей $\delta$ для $\sigma=15 \%, \sigma^{r}=1 \%$ и $a=4 \%$.

Влияние порядка аппроксимации и платежа $\delta$ на относительную ошибку проиллюстрировано на рис. 2. Как уже отмечалось, чем выше порядок, тем лучше точность. Что интересно, наблюдается хорошая устойчивость ошибки относительно платежей, вместо возрастания. Предположительно, это следствие случая $h(x)=x_{+}$: действительно, 
цена исполнения $K^{\alpha, \delta}$ увеличивается с увеличением $\delta$, и это делает опцион невыгодным для больших величин контракта (см. рис. 3). Следовательно, при росте $\delta$, все меньшие и меньшие члены дают существенный вклад в $\mathrm{VA}_{0}^{\alpha, \delta}$, что уменьшает ошибки.

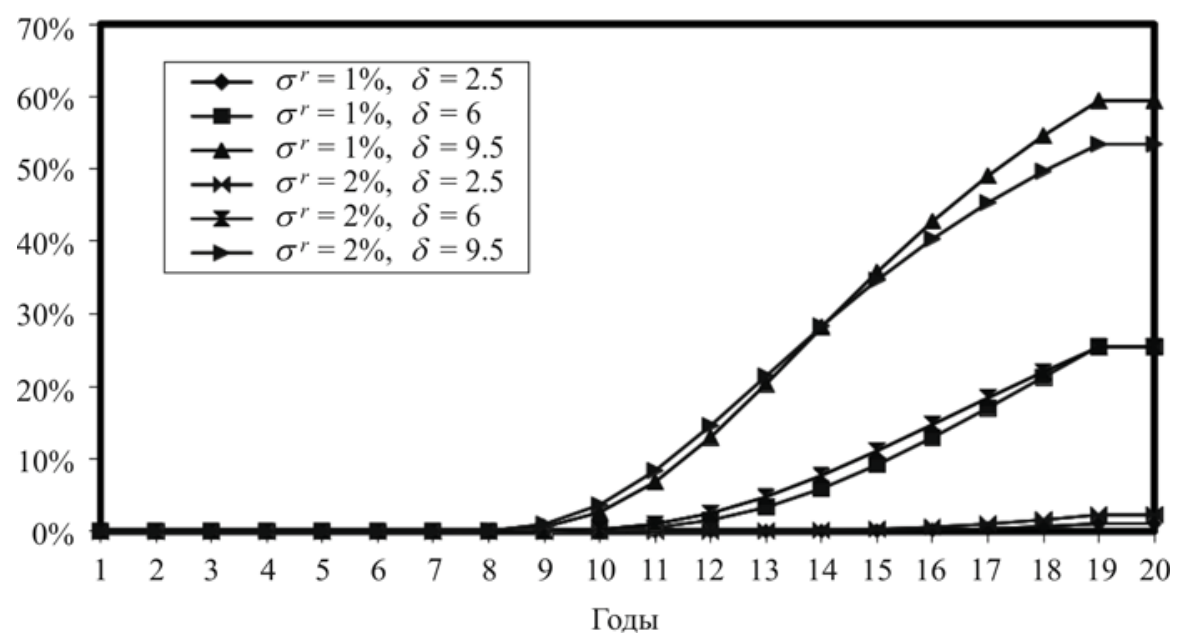

Рис. 3. Вероятность того, что счет станет отрицательным, для $\sigma=15 \%$ и $a=4 \%$.

5. Заключение. Нами найдены аппроксимационные формулы, причем в принципе любого порядка, и проанализированы их ошибки относительно выплаты контракта и волатильности модели. Учет членов третьего, четвертого или пятого порядка представляется правильным, если мы хотим иметь достаточно точные аппроксимации плавающих рент.

6. Приложение.

6.1. Доказательство леммы 1. Мы докажем только второе равенство, первое может быть получено аналогично. Доказательство проводится по индукции. Результат справедлив для $n=1$. Предположим, что он справедлив для всех $n(\geqslant 1)$ моментов платежей $\left(t_{i}\right)_{1 \leqslant i \leqslant n}$, и пусть дополнительный платеж осуществляется в момент $t_{n+1} \in\left(t_{n}, T\right]$, где мы полагаем $T=t_{n+2}$. Таким образом,

$$
\begin{aligned}
\mathrm{A}_{T}^{\alpha, \delta} & =\left(1-\alpha_{n+1}\right) \mathrm{A}_{t_{n+1}-\delta}^{\alpha,} \frac{S_{T}}{S_{t_{n+1}}}-\delta_{n+1} \frac{S_{T}}{S_{t_{n+1}}} \\
& =\left(1-\alpha_{n+1}\right) \frac{S_{T}}{S_{t_{n+1}}}\left[\pi_{0, n} S_{t_{n+1}}-\sum_{i=1}^{n} \delta_{i} \pi_{i, n} R_{t_{i}, t_{n+1}}\right]-\delta_{n+1} R_{t_{n+1}, T} \\
& =\pi_{0, n+1} S_{T}-\sum_{j=1}^{n} \delta_{j} \pi_{j, n+1} R_{t_{j}, T}-\delta_{n+1} \pi_{n+1, n+1} R_{t_{n+1}, T}
\end{aligned}
$$

откуда следует требуемый результат для случая $n+1$ момента. Лемма 1 доказана. 
6.2. Доказательство леммы 2. По формуле для облигации без купонных платежей [7, гл. 3, 5] мы имеем

$$
B(t, u)=B(0, u) \exp \left(\int_{0}^{t} r_{s} d s-\frac{1}{2} \int_{0}^{t} \Gamma^{2}(s, u) d s-\int_{0}^{t} \Gamma(s, u) d W_{s}\right)
$$

для $t \leqslant u$, откуда следует, что

$$
\begin{aligned}
\exp \left(\int_{t}^{T} r_{s} d s\right)=\frac{B(0, t)}{B(0, T)} \exp ( & \int_{0}^{T} \Gamma(s, T) d W_{s}+\frac{1}{2} \int_{0}^{T} \Gamma^{2}(s, T) d s \\
& \left.-\int_{0}^{t} \Gamma(s, t) d W_{s}-\frac{1}{2} \int_{0}^{t} \Gamma^{2}(s, t) d s\right) .
\end{aligned}
$$

С другой стороны,

$$
R_{t, T}=\exp \left(\int_{t}^{T} r_{s} d s-\frac{1}{2} \int_{t}^{T} \sigma_{s}^{2} d s+\int_{t}^{T} \sigma_{s}\left(\rho d W_{s}+\sqrt{1-\rho^{2}} d B_{s}\right)\right) .
$$

Объединяя последнее равенство с (13), мы получаем, что

$$
\begin{aligned}
R_{t, T}=\frac{B(0, t)}{B(0, T)} \exp ( & \frac{1}{2} \int_{0}^{t}\left(\Gamma^{2}(s, T)-\Gamma^{2}(s, t)\right) d s+\frac{1}{2} \int_{t}^{T}\left(\Gamma^{2}(s, T)-\sigma_{s}^{2}\right) d s \\
& +\int_{0}^{t}(\Gamma(s, T)-\Gamma(s, t)) d W_{s}+\int_{t}^{T}\left(\Gamma(s, T)+\rho \sigma_{s}\right) d W_{s} \\
& \left.+\int_{t}^{T} \sqrt{1-\rho^{2}} \sigma_{s} d B_{s}\right) .
\end{aligned}
$$

Таким образом, математическое ожидание $R_{t, T}$ равно

$$
\begin{aligned}
\frac{B(0, t)}{B(0, T)} \exp ( & \frac{1}{2} \int_{0}^{t}\left(\Gamma^{2}(s, T)-\Gamma^{2}(s, t)\right) d s+\frac{1}{2} \int_{t}^{T}\left(\Gamma^{2}(s, T)-\sigma_{s}^{2}\right) d s \\
& +\frac{1}{2} \int_{0}^{t}(\Gamma(s, T)-\Gamma(s, t))^{2} d s+\frac{1}{2} \int_{t}^{T}\left(\Gamma(s, T)+\rho \sigma_{s}\right)^{2} d s \\
& \left.+\frac{1}{2} \int_{t}^{T}\left(1-\rho^{2}\right) \sigma_{s}^{2} d s\right) .
\end{aligned}
$$

Приведение подобных членов завершает доказательство.

6.3. Доказательство утверждения 2. Достаточно рассмотреть случай $i_{1} \leqslant i_{2} \leqslant \cdots \leqslant i_{k}$. Из равенства что $D_{T}=\exp \left(-\int_{0}^{T} r_{s} d s\right)$ и соотношений (13) и (14) следует, что

$$
\begin{aligned}
c_{i_{1}, \ldots, i_{k}}=\mathbf{E}[ & B(0, T) \exp \left(-\int_{0}^{T} \Gamma(s, T) d W_{s}-\frac{1}{2} \int_{0}^{T} \Gamma^{2}(s, T) d s\right) \\
& \times \prod_{j=1}^{k} \frac{B\left(0, t_{i_{j}}\right)}{B(0, T)} \exp \left(\frac{1}{2} \int_{0}^{T} \Gamma^{2}(s, T) d s-\frac{1}{2} \int_{0}^{t_{i_{j}}} \Gamma^{2}\left(s, t_{i_{j}}\right) d s\right.
\end{aligned}
$$




$$
\begin{gathered}
\quad-\frac{1}{2} \int_{t_{i_{j}}}^{T} \sigma_{s}^{2} d s+\int_{0}^{T} \Gamma(s, T) d W_{s}-\int_{0}^{t_{i_{j}}} \Gamma\left(s, t_{i_{j}}\right) d W_{s} \\
\left.\left.\quad+\int_{t_{i_{j}}}^{T} \rho \sigma_{s} d W_{s}+\int_{t_{i_{j}}}^{T} \sqrt{1-\rho^{2}} \sigma_{s} d B_{s}\right)\right] \\
=\frac{\prod_{j=1}^{k} B\left(0, t_{i_{j}}\right)}{B(0, T)^{k-1}} \\
\times \exp \left(\frac{1}{2} \sum_{j=0}^{k} \int_{t_{i_{j}}}^{t_{i_{j+1}}}\left[(k-1) \Gamma^{2}(s, T)-j \sigma_{s}^{2}-\sum_{l=j+1}^{k} \Gamma^{2}\left(s, t_{i_{l}}\right)\right] d s\right) \\
\times \mathbf{E}\left[\operatorname { e x p } \left(\sum_{j=0}^{k} \int_{t_{i_{j}}}^{t_{i_{j+1}}}\left[(k-1) \Gamma(s, T)+j \rho \sigma_{s}-\sum_{l=j+1}^{k} \Gamma\left(s, t_{i_{l}}\right)\right] d W_{s}\right.\right. \\
\left.\left.\quad+\sum_{j=0}^{k} \int_{t_{i_{j}}}^{t_{i_{j+1}}} j \sqrt{1-\rho^{2}} \sigma_{s} d B_{s}\right)\right] .
\end{gathered}
$$

Стохастические интегралы в правой части являются винеровскими интегралами на непересекающихся интервалах, поэтому они независимы и имеют гауссовское распределение; их точное вычисление завершает доказательство.

6.4. Доказательство теоремы 2. Оно аналогично доказательству теоремы 1. Но теперь мы вместо (10) используем разложение Тейлора до второго порядка:

$$
\begin{aligned}
\mathbf{E}\left[D_{T} h\left(\mathrm{~A}_{T}^{\alpha, \delta}\right)\right]= & \mathbf{E}\left[D_{T} h\left(\bar{A}_{T}^{\alpha, \delta}\right)\right]-\sum_{i=1}^{n} \widehat{\delta}_{i} \mathbf{E}\left[D_{T}\left(R_{t_{i}, T}-\mathbf{E}\left[R_{t_{i}, T}\right]\right) h^{\prime}\left(\bar{A}_{T}^{\alpha, \delta}\right)\right] \\
& +\frac{1}{2} \mathbf{E}\left[D_{T}\left(\sum_{i=1}^{n} \widehat{\delta}_{i}\left(R_{t_{i}, T}-\mathbf{E}\left[R_{t_{i}, T}\right]\right)\right)^{2} h^{\prime \prime}\left(\bar{A}_{T}^{\alpha, \delta}\right)\right] \\
& +\operatorname{Error}_{2}(h) .
\end{aligned}
$$

По сравнению с разложением в доказательстве теоремы 1, здесь появился новый корректирующий член с коэффициентом $1 / 2$, который и надо рассмотреть:

$$
\begin{aligned}
& \mathbf{E}\left[D_{T}\left(\sum_{i=1}^{n} \widehat{\delta}_{i} R_{t_{i}, T}-K^{\alpha, \delta}\right)^{2} h^{\prime \prime}\left(\bar{A}_{T}^{\alpha, \delta}\right)\right] \\
& =\sum_{1 \leqslant i, j \leqslant n} \widehat{\delta}_{i} \widehat{\delta}_{j} \mathbf{E}\left[D_{T} R_{t_{i}, T} R_{t_{j}, T} h^{\prime \prime}\left(\bar{A}_{T}^{\alpha, \delta}\right)\right]-2 K^{\alpha, \delta} \sum_{i=1}^{n} \widehat{\delta}_{i} \mathbf{E}\left[D_{T} R_{t_{i}, T} h^{\prime \prime}\left(\bar{A}_{T}^{\alpha, \delta}\right)\right] \\
& \quad+\left(K^{\alpha, \delta}\right)^{2} \mathbf{E}\left[D_{T} h^{\prime \prime}\left(\bar{A}_{T}^{\alpha, \delta}\right)\right]=: \tau_{1}+\tau_{2}+\tau_{3} .
\end{aligned}
$$

Преобразуем каждое из трех слагаемых.

Член $\tau_{1}$. Мы используем замену меры $\mathbf{Q}_{t_{i}, t_{j}}$ : в силу утверждения 3 $\bar{A}_{T}^{\alpha, \delta}$ относительно $\mathbf{Q}_{t_{i}, t_{j}}$ распределена так же, как $\pi_{0, n}\left(c_{0, i, j} / c_{i, j}\right) \widetilde{S}_{T}-K^{\alpha, \delta}$ 
относительно $\mathbf{Q}$, откуда следует, что

$$
\begin{aligned}
\tau_{1} & =\sum_{1 \leqslant i, j \leqslant n} \widehat{\delta}_{i} \widehat{\delta}_{j} c_{i, j} \mathbf{E}_{\mathbf{Q}_{t_{i}, t_{j}}}\left[h^{\prime \prime}\left(\bar{A}_{T}^{\alpha, \delta}\right)\right] \\
& =\sum_{1 \leqslant i, j \leqslant n} \widehat{\delta}_{i} \widehat{\delta}_{j} c_{i, j} \mathbf{E}\left[h^{\prime \prime}\left(\pi_{0, n} \frac{c_{0, i, j}}{c_{i, j}} \widetilde{S}_{T}-K^{\alpha, \delta}\right)\right] \\
& =\left.\sum_{1 \leqslant i, j \leqslant n} \widehat{\delta}_{i} \widehat{\delta}_{j} c_{i, j} \partial_{K}^{2} \mathbf{E}\left[h\left(\pi_{0, n} \frac{c_{0, i, j}}{c_{i, j}} \widetilde{S}_{T}-K\right)\right]\right|_{K=K^{\alpha, \delta}} .
\end{aligned}
$$

Членьл $\tau_{2} u \tau_{3}$. Аналогично, мы имеем

$$
\begin{aligned}
\tau_{2} & =-\left.2 K^{\alpha, \delta} \sum_{i=1}^{n} \widehat{\delta}_{i} c_{i} \partial_{K}^{2} \mathbf{E}\left[h\left(\pi_{0, n} \frac{c_{0, i}}{c_{i}} \widetilde{S}_{T}-K\right)\right]\right|_{K=K^{\alpha, \delta}}, \\
\tau_{3} & =\left.\left(K^{\alpha, \delta}\right)^{2} B(0, T) \partial_{K}^{2} \mathbf{E}\left[h\left(\frac{\pi_{0, n}}{B(0, T)} \widetilde{S}_{T}-K\right)\right]\right|_{K=K^{\alpha, \delta}} .
\end{aligned}
$$

Теорема 2 доказана.

6.5. Доказательство теоремы 3. Мы проведем доказательство только для $\operatorname{Error}_{1}(h)$ (т.е. для $k=1$ ), случай $\operatorname{Error}_{2}(h)$ рассматривается аналогично. Для краткости будем писать $A \leqslant c$ вместо $A \leqslant c B$, где $c$ - некоторая положительная константа, которая остается равномерно ограниченной при стремлении параметров модели к нулю и может меняться в процессе вычислений. Как обычно, $\|Z\|_{p}$ обозначает $L_{p}$-норму случайной величины $Z$. Положим $\bar{\sigma}^{r}:=\sup _{0 \leqslant t \leqslant T}\left|\sigma_{t}^{r}\right|$ и $\bar{\sigma}:=\sup _{0 \leqslant t \leqslant T}\left|\sigma_{t}\right|$. Из (9) и (10) следует, что

$$
\operatorname{Error}_{1}(h)=\left[\mathbf{E} \int_{0}^{1} D_{T}\left(\sum_{i=1}^{n} \widehat{\delta}_{i}\left(R_{t_{i}, T}-\mathbf{E}\left[R_{t_{i}, T}\right]\right)\right)^{2}(1-\lambda) h^{\prime \prime}\left(A_{T}^{\lambda}\right) d \lambda\right],
$$

где $A_{T}^{\lambda}=\bar{A}^{\alpha, \delta}-\lambda \sum_{i=1}^{n} \widehat{\delta}_{i}\left(R_{t_{i}, T}-\mathbf{E}\left[R_{t_{i}, T}\right]\right)$. Отсюда, из неравенства Гёльдера и стандартных оценок $L_{p}$-норм $A_{T}^{\lambda}$ мы получаем, что

$$
\left|\operatorname{Error}_{1}(h)\right| \leqslant_{c} C_{h}\left(1+S_{0}^{p_{h}}\right)\left(n \sup _{1 \leqslant i \leqslant n} \widehat{\delta}_{i}\left\|R_{t_{i}, T}-\mathbf{E}\left[R_{t_{i}, T}\right]\right\|_{6}\right)^{2} .
$$

Остается оценить сверху величину $\left\|R_{t_{i}, T}-\mathbf{E}\left[R_{t_{i}, T}\right]\right\|_{6}$ для $1 \leqslant i \leqslant n$. Из (14) вытекает равенство

$$
\begin{aligned}
R_{t_{i}, T}-\mathbf{E}\left[R_{t_{i}, T}\right]= & \frac{B\left(0, t_{i}\right)}{B(0, T)} \exp (A) \exp \left(\frac{1}{2}\langle N\rangle_{T}\right) \\
& \times\left(\exp \left(N_{T}-\frac{1}{2}\langle N\rangle_{T}\right)-1\right),
\end{aligned}
$$

где

$$
A=\frac{1}{2} \int_{0}^{T} \Gamma^{2}(s, T) d s-\frac{1}{2} \int_{0}^{t_{i}} \Gamma^{2}\left(s, t_{i}\right) d s-\frac{1}{2} \int_{t_{i}}^{T} \sigma_{s}^{2} d s
$$


и

$$
\begin{aligned}
N_{u}= & \int_{0}^{u}\left(\Gamma(s, T)-\Gamma\left(s, t_{i}\right) \mathbf{1}_{s \leqslant t_{i}}+\rho \sigma_{s} \mathbf{1}_{s \geqslant t_{i}}\right) d W_{s} \\
& +\int_{0}^{u} \sqrt{1-\rho^{2}} \sigma_{s} \mathbf{1}_{s \geqslant t_{i}} d B_{s}
\end{aligned}
$$

для $0 \leqslant u \leqslant T$. Из [11, лемма 4.3$]$ следует (для $p \geqslant 1$ ), что

$$
\left\|e^{J_{T}}-1\right\|_{p} \leqslant\left\|J_{T}\right\|_{2 p} e^{2 p\langle N\rangle_{T}}
$$

где $J_{T}=N_{T}-\langle N\rangle_{T} / 2$. Таким образом,

$$
\left\|R_{t_{i}, T}-\mathbf{E}\left[R_{t_{i}, T}\right]\right\|_{6} \leqslant \frac{B\left(0, t_{i}\right)}{B(0, T)} \exp (A) \exp \left(\frac{1}{2}\langle N\rangle_{T}\right)\left\|J_{T}\right\|_{12} \exp \left(12\langle N\rangle_{T}\right) .
$$

Используя стандартные вычисления, находим, что

$$
\begin{aligned}
\langle N\rangle_{T}= & \int_{0}^{t_{i}}\left(\Gamma(s, T)-\Gamma\left(s, t_{i}\right)\right)^{2} d s+\int_{t_{i}}^{T}\left(\Gamma(s, T)+\rho \sigma_{s}\right)^{2} d s \\
& +\int_{t_{i}}^{T}\left(1-\rho^{2}\right) \sigma_{s}^{2} d s \\
\leqslant & 2\left(\int_{0}^{T} \Gamma^{2}(s, T) d s+\int_{0}^{t_{i}} \Gamma^{2}\left(s, t_{i}\right) d s+\bar{\sigma}^{2}\left(T-t_{i}\right)\right) \\
\leqslant & \left(\bar{\sigma}^{r}\right)^{2} T \min \left(T^{2}, a^{-2}\right)+\bar{\sigma}^{2}\left(T-t_{i}\right) .
\end{aligned}
$$

Отсюда, из (16) и неравенств Буркхольдера-Дэвиса-Ганди [20, гл. IV, раздел 4] вытекает оценка

$$
\left\|R_{t_{i}, T}-\mathbf{E}\left[R_{t_{i}, T}\right]\right\|_{6} \leqslant c \bar{\sigma}^{r} \sqrt{T} \min \left(T, a^{-1}\right)+\bar{\sigma} \sqrt{T-t_{i}} .
$$

Доказательство завершается подстановкой полученного неравенства B (15).

\section{СПИСОК ЛИТЕРАТУРЫ}

1. Bauer D., Bergmann D., Kiesel R. On the risk-neutral valuation of life insurance contracts with numerical methods in view. - ASTIN Bull., 2010, v. 40, № 1, p. 6595.

2. Ben Zineb T. Méthodes numériques efficaces pour la valorisation des GMWB. Ph.D. thesis. Paris: Ecole Polytechnique.

3. Bompis R., Gobet E. Asymptotic and non asymptotic approximations for option valuation. - Recent Developments in Computational Finance: Foundations, Algorithms and Applications. Ed. by T. Gerstner and P. Kloeden, Hackensack: World Scientific, 2012, p. 159-241.

4. Ben Zineb T., Gobet E. Preliminary control variates to improve empirical regression methods. - Monte-Carlo Methods and Appl., 2013, v. 19, № 4, p. 331-354. 
5. Bauer D., Kling A., Russ J. A universal pricing framework for guaranteed minimum benefits in variable annuities. - ASTIN Bull., 2008, v. 38, № 2, p. 621-651.

6. Biffis E., Millossovich P. The fair value of guaranteed annuity options. - Scand. Actuar. J., 2006, v. 1, № 1, p. 23-41.

7. Brigo D., Mercurio F. Interest Rate Models: Theory and Practice. Berlin: Springer, 2006, $981 \mathrm{p}$.

8. Bacinello A.R., Millossovich P., Olivieri A., Pitacco E. Variable annuities: a unifying valuation approach. - Insurance Math. Econom., 2011, v. 49, № 3, p. 285-297.

9. Chen Z., Forsyth P.A. A numerical scheme for the impulse control formulation for pricing variable annuities with a guaranteed minimum withdrawal benefit (GMWB). - Numer. Math., 2008, v. 109, № 4, p. 535-569.

10. Dai M., Kwok Y.K., Zong J. Guaranteed minimum withdrawal benefit in variable annuities. - Math. Finance, 2008, v. 18, № 4, p. 595-611.

11. Étoré P., Gobet E. Stochastic expansion for the pricing of call options with discrete dividends. - Appl. Math. Finance, 2012, v. 19, № 3, p. 233-264.

12. Friz P.K., Gatheral J., Gulisashvili A., Jacquier A., Teichmann J. (eds.), Large Deviations and Asymptotic Methods in Finance. Berlin: Springer-Verlag, 2015, 590 p.

13. Glasserman P. Monte Carlo methods in Financial Engineering. New York: Springer Verlag, 2004, 596 p.

14. Gerber H. U., Shiu E.S.W., Yang H. Valuing equity-linked death benefits and other contingent options: a discounted density approach. - Insurance Math. Econom., 2012, v. 51, № 1, p. 73-92.

15. Ledlie M.C., Corry D.P., Finkelstein G.S., Ritchie A.J., Su K., Wilson D.C.E. Variable annuities. - British Actuar. J., 2008, v. 14, № 2, p. 327-389.

16. Møller T. Risk-minimizing hedging strategies for unit-linked life insurance contracts. - ASTIN Bulletin, 1998, v. 28, № 1, p. 17-47.

17. Møller T. Risk-minimizing hedging strategies for insurance payment processes. Finance Stoch., 2001, v. 5, № 4, p. 419-446.

18. Musiela M., Rutkowski M. Martingale Methods in Financial Modelling. Berlin: Springer, 2005, $636 \mathrm{p}$.

19. Milevsky M.A., Salisbury T.S. Financial valuation of guaranteed minimum withdrawal benefits. - Insurance Math. Econom., 2006, v. 38, № 1, p. 21-38.

20. Revuz D., Yor M. Continuous Martingales and Brownian Motion. Berlin: Springer, 1999, 602 p.

21. Ulm E. R. Analytic solution for return of premium and rollup guaranteed minimum death benefit options under some simple mortality laws. - ASTIN Bull., 2008, v. 38, № 2 , p. 543-563.

22. Vanduffel S., Shang Z., Henrard L., Dhaene J., Valdez E. A. Analytic bounds and approximations for annuities and Asian options. - Insurance Math. Econom., 2008, v. 42 , № 3, p. 1109-1117. 